\title{
Diagnostic Significance of Serum Total Bile Acid Levels in Suspected Cases of Obstetric Cholestasis
}

${ }^{1}$ Hijab Batool, ${ }^{1}$ Muhammad Dilawar Khan, ${ }^{2}$ Omar Rasheed Chughtai, ${ }^{1}$ Shakeel Ashraf

${ }^{I}$ Department of Clinical Chemistry and Immunology, Chughtai Institute of Pathology, Lahore

${ }^{2}$ Director Operations, Chughtai Institute of Pathology, Lahore

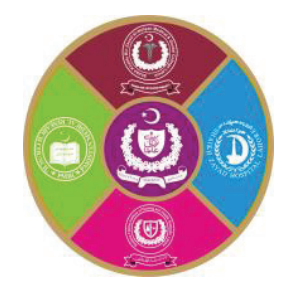

\begin{abstract}
Introduction: Intrahepatic Cholestasis of Pregnancy (ICP) is significantly associated with adverse maternal and fetal outcome and therefore accurate diagnosis and timely initiation of therapy is of utmost importance.

Aims \& Objectives: To find out the diagnostic significance of serum total bile acid measurement in suspected cases of obstetric cholestasis. Place and duration of study: It was a cross sectional study conducted in the Department of Clinical Chemistry and Immunology, Chughtai Institute of Pathology from July 2018 to February 2019. Material \& Methods: Two hundred pregnant females whose serum total bile acid (TBA) was determined were included in the study. Pregnant females with cholestasis due to medical reasons other than ICP were excluded from the study. The patients were contacted for relevant clinical details which were recorded on a pre-designed proforma. Data was analyzed using SPSS 23.0. Univariate analysis was done to find out percentages and frequencies. Results: Mean age of 200 pregnant females was 27 years having Mean TBA value of $32.8 \mu \mathrm{mol} / \mathrm{L}$. $63 \%$ females were primiparous. 197 (98.5\%) patients gave the history of pruritus while 193 (96.5\%) patients presented with dark urine. Only 30 patients (15\%) gave history of using progesterone during pregnancy. Total Bile acid value was $>10 \mu \mathrm{mol} / \mathrm{L}$ for 84 primiparous and 48 multiparous cases $(n=132)$. There was no significant difference in the mean TBA value on the basis of parity, medication, mode of delivery or presence and absence of itch. Conclusion: ICP is a multi factorial reversible condition and bile acid estimation plays a major role in correct clinical assessment of patients with obstetric cholestasis.
\end{abstract}

ABSTRACT

Key words: Total Bile Acid, Obstetric Cholestasis, pruritus, jaundice

\section{INTRODUCTION}

$\mathrm{B}_{\mathrm{ilt}}$ acids are synthesized in the liver from cholesterol as precursor. Primary bile acids are cholic and chenodeoxycholic acid which are metabolized in the intestinal lumen by action of bacterial enzyme 7- $\alpha$-dehydroxylase. As a result of this metabolism secondary bile acids (deoxycholic and lithocholicacid) and a fraction of tertiary bile acid (ursodeoxycholicacid) are formed. Glycine and taurine react with the bile acids to form conjugated bile acids. The term "Total Bile Acids" refer to primary, secondary and tertiary bile acids along with their conjugates. ${ }^{1}$

Bile is secreted by the liver and stored in the gallbladder of which bile acids are a major constituent. More than $90 \%$ of the bile acids which pass through GIT are reabsorbed from small intestine via enterohepatic circulation, processed in the liver and re-secreted into the gall bladder.
Impairment of enterohepatic circulation leads to increased level of bile acids in the serum and urine. ${ }^{2}$ Metabolically bile acids have several important roles. These are the primary metabolic pathway for cholesterol catabolism, aid in digestion specifically lipid and lipid soluble vitamins and play a role in glucose and lipoprotein metabolism. ${ }^{3}$ Bile acids are increased after food ingestion therefore a fasting sample is recommended. ${ }^{4}$ Within a period of 24 hours, liver removes about 20 grams of bile salts from the circulation and abnormal bile acid levels indicate presence of liver disease. ${ }^{4,5}$

There can be many potential sites for the primary or secondary disturbances of bile acid levels. Hepatocyte dysfunction can result in decreased level of primary bile acid with a decreased primary to secondary bile acid ratio. In the non-fasting state, there is decreased extraction from plasma leading to increased concentration of bile acids. On the other hand, in cholestatic disorders, there is increased concentration of total bile acids with increased 
primary to secondary bile acid ratio. In intestinal disorders, there is low concentration of both primary and secondary bile acids due to increased fecal loss. ${ }^{1}$ Moreover, increase in total bile acid level can also be seen in hepatic coma, alcoholic liver disease, chemical and drug induced liver damage, liver cirrhosis and cystic fibrosis. ${ }^{6}$ Some of the studies in animals have shown that bile acids may damage pancreatic cells and can lead to pancreatitis. ${ }^{7}$

The prevalence of ICP is higher in South Asian and South American populations as compared to European countries. ${ }^{8}$ According to a survey conducted in 2014 on 1000 obstetric patients in Pakistan, the frequency of patients having ICP was $3.1 \% .^{9}$ Bile acid estimation is used as a marker for hepatic integrity and aids in the diagnosis of obstetric cholestasis which is one of the commonest causes of liver disease during gestational period. Factors such as advanced maternal age, previous history of ICP, positive family history, multiple pregnancies and use of oral contraceptive pills are found to be associated with increased risk of ICP. ${ }^{10}$, ${ }^{11}$ The genetic predisposition becomes clear by the evidence of family clustering, ethnic variations and discovery of mutations in the genes encoding for hepatobiliary transport proteins. ${ }^{12}$ Intrahepatic Cholestasis of pregnancy (ICP) is characterized by pruritus (unexplained) with increased level of serum bile acids and/or abnormal liver function tests in the second or third trimester of pregnancy. Abnormal laboratory picture and pruritus occurs in the absence of other liver diseases and there is resolution of all the signs and symptoms within 2 to 3 weeks after delivery of the neonate. ${ }^{13,14}$ ICP is significantly associated with adverse maternal and fetal outcome and therefore accurate diagnosis and timely initiation of therapy is of utmost importance. Bile acid estimation plays a major role in correct clinical assessment of patients with obstetric cholestasis. ${ }^{5}$

Non availability of serum bile acid analysis is a major limitation in majority of the studies and in many clinical settings the diagnosis of ICP is made by exclusion of other medical conditions and clinical features. Assessment of the diagnostic accuracy of TBA may help the clinicians improve the approach towards the disease directly benefit outcome of pregnant females and their newborns. The range of values used to assess pregnant females is same as that of non pregnant population in most of the clinical laboratories i.e. $0-10 \mu \mathrm{mol} / \mathrm{L} .{ }^{15}$

\section{MATERIAL AND METHODS}

It was a cross sectional study conducted on pregnant females whose total bile acid test was performed.
After obtaining permission from Institutional Ethical Review Board, pregnant females fulfilling the inclusion criteria whose serum TBA were performed from July 2018 (since the test was included in the panel) to February 2019 were included in the study $(n=200)$ The patients were contacted for history and any relevant clinical details. History along with mode of delivery and neonatal outcome (only if patient had delivered at the time of study) were recorded on a pre-designed proforma.

Serum Total Bile acids were analyzed on Architect ci8200 by isoenzymatic cycling colorimetric method where bile acids in the sample are converted to their corresponding oxosteroids in the presence of excess $\mathrm{NADH}$ (nicotinamide adenine dinucleotide) and thio-NAD ${ }^{+}$(thionicotinamide adenine dinucleotide). For quality assessment controls were run on a daily basis.

\section{Statistical analysis:}

The results were analyzed statistically using SPSS 23.0. A univariate analysis was done using percentages, frequencies and means. Mann-Whitney test was used to find out difference between mean Serum TBA among different groups. A p value of $<0.05$ was considered significant.

\section{RESULTS}

Mean age of patients was $27 \pm 4$ years (Minimum age 18 years Maximum age 39 years). Mean TBA value was $32.8 \mu \mathrm{mol} / \mathrm{L}$ (IQR 23.88, Minimum 2.10 $\mu \mathrm{mol} / \mathrm{L}$-Maximum $346.80 \mu \mathrm{mol} / \mathrm{L}$ ). Out of all the 200 pregnant females, $126(63 \%)$ were primiparous and $74(37 \%)$ females were multiparous. In our study, 197 (98.5\%) females gave the history of pruritus whereas only $3(1.5 \%)$ females did not have any pruritus (TBA value was less than $10 \mu \mathrm{mol} / \mathrm{L}$ ) and they only requested the test because they were advised by their consultants. 193 (96.5\%) females presented with dark urine whereas only $7(3.5 \%)$ females did not give history of dark colored urine. 30 females $(15 \%)$ gave history of using progesterone during their pregnancy as prescribed by their gynecologist and stated that they developed pruritus right after starting the medicine. 44(22\%) females gave birth to the neonate via spontaneous vaginal delivery(SVD). 127 (63.5\%) females had Caesarean Section (C-section) while 29 (14.5\%) females were still pregnant at the time of study. Among the multiparous females, 12 females who gave birth vaginally in their previous pregnancies had to undergo C-section due to threatened complications. Total Bile acid value was more than 
$10 \mu \mathrm{mol} / \mathrm{L}$ for 84 primiparous and 48 multiparous females $(n=132)$. Among the 30 females who took progesterone in their pregnancy, 17 had TBA value more than $10 \mu \mathrm{mol} / \mathrm{L}$. There was no significant difference between the TBA value among primiparous and multiparous women. Similarly, the women who took progesterone during their pregnancy had mean TBA value of $24.1 \mu \mathrm{mol} / \mathrm{L}$, which was not significantly different from the women who did not take progesterone. Mean TBA value for women who underwent $\mathrm{C}$-Section was $32.8 \mu \mathrm{mol} / \mathrm{L}$ and $40.4 \mu \mathrm{mol} / \mathrm{L}$ for women who had a normal vaginal delivery. There was no significant difference in mean serum total bile acid levels in various groups of patients ( $p$-value $>0.05$ MannWhitney test) (Table-1).

\begin{tabular}{|c|c|c|}
\hline Groups & $\begin{array}{c}\text { Mean TBA } \\
\text { Value }(\mu \mathrm{mol} / \mathrm{L})\end{array}$ & P value \\
\hline Primiparous females & 35.6 & \multirow{2}{*}{0.340} \\
\hline Multiparous females & 32.4 & \\
\hline Women who took progesterone & 24.1 & \multirow{2}{*}{0.341} \\
\hline $\begin{array}{l}\text { Women who did not take } \\
\text { progesterone }\end{array}$ & 34.2 & \\
\hline $\begin{array}{l}\text { Woman who delivered via } \\
\text { spontaneous vaginal delivery }\end{array}$ & 40.4 & \multirow{2}{*}{0.395} \\
\hline $\begin{array}{l}\text { Woman who delivered via C } \\
\text { Section }\end{array}$ & 32.8 & \\
\hline Woman presented with itching & 32.7 & \multirow{2}{*}{0.786} \\
\hline $\begin{array}{l}\text { Woman with no history of } \\
\text { itching }\end{array}$ & 27.4 & \\
\hline
\end{tabular}

Table-1: Serum Total Bile Acid levels in various groups of patients $(n=200)$

\section{DISCUSSION}

ICP appear as a hepatic disorder in pregnancy and is the second most common cause of jaundice in pregnancy is ICP following viral hepatitis. ${ }^{16,17} \mathrm{~A}$ combination of multiple factors is responsible for the development of obstetric cholestasis including environmental and hormonal factors acting in conjugation with genetic tendency. ${ }^{18}$

A multiethnic study conducted in United Kingdom revealed that the incidence of ICP in multiethnic population was $0.7 \%$ where as in Indian and Pakistani population this incidence was 1.2 to $1.5 \% .{ }^{19}$

In an Indian study the diagnosis of ICP was made only on the basis of liver function tests and clinical features of patients and the authors concluded that in the absence of liver function tests, aminotransferases can aid in the diagnosis of ICP. ${ }^{20}$ More than $90 \%$ of the females in our study gave history of pruritus. A Pakistani study showed similar results in which pruritus was present in $85 \%$ of the patients. ${ }^{9}$ Majority of the patients $(63 \%)$ had delivered via $\mathrm{C}$ section in our study and the results are similar to the above stated study in which $58 \%$ of the patients had C-section. ${ }^{9}$

A systematic review published in 2019 stated that a cut off value of $10 \mathrm{umol} / \mathrm{L}$ for TBA had an overall sensitivity ranging from $72 \%$ to $98 \%$ and specificity ranging from $81 \%$ to $97 \% .{ }^{21} \mathrm{~A}$ retrospective study conducted in China revealed that pregnant females had increased fasting serum bile acid levels even without the presence of symptoms and these asymptomatic pregnant females had similar or a higher risk of still birth than patients with ICP. ${ }^{22}$ Demir along with his colleagues while establishing relationship between Serum Bile Acid levels and maternal and fetal outcomes found that mean TBA level in preterm birth group was significantly higher as compared to the term birth group. ${ }^{23}$ In another study determining the role of bile acid ratio of cholic acid to chenodeoxycholic acid in diagnosis of ICP stated that the ratio contributed to the diagnosis of ICP and use of Liver Function tests in the diagnosis of ICP with bile acid ratios decreased the percentage of positive results. ${ }^{24}$ Yannik Bacq in 2014 concluded in his study that Serum total Bile acid levels can be used to diagnose cases of ICP and can also be used as a followup investigation. ${ }^{25}$

Some studies have shown that TBA level may rise up to 25 fold in suspected cases of obstetric cholestasis. Also there can be a marked rise in the ratio of cholic and chenodeoxycholic acid in ICP. A triad of increased TBA, elevated cholic acid level and a decreased glycine to taurine ratio can be used to exclude other causes and aid in differential diagnosis. Elevated ALT, AST and mild hyperbilirubinemia (Rarely $>6 \mathrm{mg} / \mathrm{dl}$ ) may further help to reach a final diagnosis. ${ }^{8}$ Other investigations which might help in timely diagnosis are plasma glucose levels and clotting profile of the patient. ${ }^{17} \mathrm{In}$ the absence of bile acids, obstetric cholestasis may be diagnosed with the help of clinical findings and elevated transaminases, provided both of these resolve after delivery. ${ }^{26}$

Pregnancy specific reference ranges are necessary for proper interpretation of results. Egan along with his colleagues conducted a study in which he proposed reference ranges of total bile acid in pregnancy in relation with gestational age. The authors found that value of serum bile acids ranged from 0.3 to $9.8 \mu \mathrm{mol} / \mathrm{L}$, which is considerably different from the range given for non-pregnant patients. $^{2}$ Another Chinese study found the mean TBA value raised from $24^{\text {th }}$ gestational week (range 
$0.3-8.6 \mu \mathrm{mol} / \mathrm{L})$ and reached its maximum at $36^{\text {th }}$ gestational week with a mean of $2.97 \mu \mathrm{mol} / \mathrm{L}$ (range $0.5-12.1 \mu \mathrm{mol} / \mathrm{L}) .{ }^{27}$ Lee et al conducted a study to find out Bile acid concentration reference ranges in Latin pregnant females and found a range of $<8.5$ $\mu \mathrm{mol} / \mathrm{L} .^{28}$ Non availability of an established reference range of TBA in pregnancy is a major limitation in accurate diagnosis of ICP.

It is very important to create clinical awareness among the general population regarding ICP. Early diagnosis with timely initiation of therapy is important for improved fetal prognosis in ICP. Availability of bile acid testing in clinical settings can be very helpful in this regard. Due to nonavailability of this test in many clinical settings of Pakistan, the diagnosis of ICP is based on exclusion. Further a reference range of TBA according to gestational age should be established. This pregnancy related TBA reference range will be helpful to physicians for correct diagnosis of ICP.

\section{CONCLUSION}

In our study Serum Total Bile Acid measurement provided valuable clinical information while diagnosing ICP. Serum Total Bile Acid was raised in cases of Intra hepatic Cholestasis of Pregnancy.

\section{Conflict of interest:}

In accordance with our ethical obligation as researchers we are declaring that Omar Rasheed Chughtai is Director Operations at Chughtai Institute of Pathology.

\section{Limitations:}

Our study is a single centered study with limited sample size. Further supporting studies from multiple clinical settings with larger sample size are suggested to provide more clinical evidence relating to diagnostic accuracy of Serum Total Bile Acids. Our desire was to share the findings to create a parameter which can be replicated in other studies.

\section{REFERENCES}

1. Burtis CA, Ashwood ER, Bruns DE. Tietz Textbook of Clinical Chemistry and Molecular Diagnostics. 5th ed. St. Louis, MO: Elsevier Saunders. 2012; 1641-43.

2. Egan N, Bartels A, Khashan A, et al. Reference standard for serum bile acids in pregnancy. BJOG. 2012; 119:493-8.

3. deAguiarVallim TQ, Tarling EJ, Edwards PA, Pleiotropic roles of bile acids in metabolism. Cell Metab. 2013; 17:657-69.
4. McPherson RA, Pincus MR. Henry's Clinical Diagnosis and Management by Laboratory Methods. $21^{\text {st }}$ d. Philadelphia, PA: Elsevier; 2007; 266-7.

5. Hofmann AF, Hagey LR. Key discoveries in bile acid chemistry and biology and their clinical applications: history of the last eight decades. J Lipid Res. 2014; 55:1553-95.

6. Maleszka A, Dumnicka P, Matuszyk A, Pędziwiatr M, Mazur-Laskowska M, Sporek M et al. The Diagnostic Usefulness of Serum Total Bile Acid Concentrations in the Early Phase of Acute Pancreatitis of Varied Etiologies. International Journal of Molecular Sciences. 2017; 18(1):106.

7. Venglovecz V, Rakoczay Z, Hegyi P. The effects of bile acids on pancreatic ductal cells. Panceapedia. 2012.

8. Ozkan S, Ceylan Y, Ozkan O, Yildirim S. Review of a challenging clinical issue: Intrahepatic cholestasis of pregnancy. World Journal of Gastroenterology.2015;21(23):7134-41

9. Hafeez M, Ansari A, Parveen S, Salamat A, Aijaz A. Frequency of intrahepatic cholestasis of pregnancy in Punjab Pakistan: A single centre study. JPak Med Assoc.2016;66(2):203-6

10. Lee NM, Brady CW .Liver disease in pregnancy. World J Gastroenterol. 2009 Feb 28; 15(8):897-906.

11. Williamson $\mathrm{C}$, Geenes V. Intrahepatic cholestasis of pregnancy. Obstet Gynecol. 2014 Jul; 124(1):120-33.

12. Anzivino C, Odoardi MR, Meschiari E, Baldelli E, Facchinetti F, Neri I et al. ABCB4 and ABCB11 mutations in intrahepatic cholestasis of pregnancy in an Italian population. Dig Liver Dis. 2013 Mar; 45(3):226-32.

13. Lammert F, Marschall HU, Glantz A, Matern S. Intrahepatic cholestasis of pregnancy: molecular pathogenesis, diagnosis and management. J Hepatol.2000; 33:1012-21.

14. Beuers U, Pusl T. Intrahepatic cholestasis of pregnancy-a heterogeneous group of pregnancy related disorders. Hepatology. 2006; 43:647-9.

15. Geenes V, Williamson C. Intrahepatic cholestasis of pregnancy. World J Gastroenterol 2009; 15:2049-66.

16. Diken Z, Usta IM, Nassar AH. A clinical approach to intrahepatic cholestasis of pregnancy. Am J Perinatol. 2014 Jan; 31(1):1-8.

17. Saleh MM, Abdo KR J. Intrahepatic cholestasis of pregnancy: review of the literature and evaluation of current evidence. Women's Health (Larchmt). 2007; 16(6):833-41. 
18. Than NN, Neuberger J. Liver abnormalities in pregnancy. Best Pract Res ClinGastroenterol. 2013; 27(4):565-75.

19. Madazli R, Yuksel MA, Oncul M, Tuten A, Guralp O, Aydin B. Pregnancy outcomes and prognostic factors in patients with intrahepatic cholestasis of pregnancy. J ObstetGynaecol 2015; 35:358-61.

20. Singh G, Sidhu K. Cholestasis of Pregnancy: A Prospective Study. Medical Journal Armed Forces India. 2008; 64(4):343-345.

21. Manzotti C, Casazza G, Stimac T, Nikolova D, Gluud C. Total serum bile acids or serum bile acid profile, or both, for the diagnosis of intrahepatic cholestasis of pregnancy. Cochrane Database of Systematic Reviews 2019, Issue 7.

22. Feng D, He W. Asymptomatic elevated total serum bile acids representing an unusual form of intrahepatic cholestasis of pregnancy. International Journal of Gynecology \& Obstetrics. 2016; 134(3):343-344.

23. Demir B, Güneş E, Atalay M. Intrahepatic cholestasis of pregnancy: Relationship between bile acid levels and maternal and fetal complications. Journal of Turkish Society of Obstetric and Gynecology. 2014;11(3):148-152.

24. Huang W, Gowda M, Donnelly J. Bile Acid Ratio in Diagnosis of Intrahepatic Cholestasis of Pregnancy. American Journal of Perinatology. 2008; 26(04):291-294.

25. Bacq Y, Sentilhes L. Intrahepatic cholestasis of pregnancy: Diagnosis and management. Clinical Liver Disease. 2014; 4(3):58-61.

26. Geenes V, Williamson C, Chappell L. Intrahepatic cholestasis of pregnancy. The Obstetrician \&Gynaecologist.2016;18(4):273-81

27. Chen L, Wen H, Zhu B, Qian Y, He J. Reference standard of total bile acid concentration of Chinese pregnant women: analyses of 11022 Chinese pregnant women. Int J ClinExp Med. 2018; 11(7):7312-7318.

28. Lee HR, Ouzounian J, Goodwin T, Incerpi M, Miller D, Zhang K, Caulfield M et al. Bile Acid Concentration Reference Ranges in a Pregnant Latina Population. Amer J Perinatol 2013; 30(05): 389-394.

\section{The Authors:}

Dr. Hijab Batool

Resident Pathologist,

Department of Clinical Chemistry and Immunology,

Chughtai Institute of Pathology, Lahore

Prof. Muhammad Dilawar Khan

Professor of Chemical Pathology,

Department of Clinical Chemistry and Immunology,

Chughtai Institute of Pathology, Lahore

Dr. Omar Rasheed Chughtai

Assistant Professor of Histopathology,

Director Operations,

Chughtai Institute of Pathology, Lahore

Shakeel Ashraf

Technical Supervisor,

Department of Clinical Chemistry and Immunology,

Chughtai Institute of Pathology, Lahore

\section{Corresponding Author:}

Dr. Hijab Batool

Resident Pathologist,

Department of Clinical Chemistry and Immunology,

Chughtai Institute of Pathology, Lahore

E-mail: batool.hijab@gmail.com 